\title{
The assessment of visuo-spatial neglect after acute stroke
}

\author{
S P Stone, B Wilson, A Wroot, P W Halligan, L S Lange, J C Marshall, R J Greenwood
}

\begin{abstract}
Forty four consecutive patients with acute hemispheric stroke and forty seven elderly controls with no neurological disease were assessed for visuo-spatial neglect, using a modified neglect test battery. Neglect was found to be equally common in patients with right hemisphere and left hemisphere stroke three days after stroke $(72 \%$ versus $62 \%)$. It was more severe in those with a right hemisphere stroke and resolved more frequently in those with a left hemisphere stroke. The battery was validated against an occupational therapist's assessment of neglect on self-care tasks. The inter-observer reliability was good and it was possible to monitor changes over time with the battery.
\end{abstract}

Visuo-spatial neglect is an important predictor of poor outcome after stroke. ${ }^{1-5}$ The natural history of neglect after stroke, however, remains unclear. Estimates of the frequency of visuo-spatial neglect $(33-85 \%$ in right and $0-25 \%$ in left hemisphere strokes) and of the frequency of recovery $(0-50 \%$ in right and $60 \%$ in left hemisphere stroke) vary widely. ${ }^{2-4} 6-10$

The variation in these estimates is the result, firstly, of the use of different tests to detect visuo-spatial neglect and, secondly, of the use of only one or two tests chosen often on the basis of their simplicity. This practise is unsatisfactory, because clinical neglect is not an all or none phenomenon. ${ }^{11}{ }^{12}$ Neglect is not observed in all activities or all clinical tests at any one time. ${ }^{13}$ It cannot therefore be assumed that all tests assess or measure the same neuropsychological variable or mechanism. ${ }^{1415}$

Over 50 bedside tests of visuo-spatial neglect have been used in various combinations. The tests most commonly used are probably line cancellation and drawing tasks. ${ }^{4616}$ Recently, Wilson et al developed the Behavioural Inattention Test (BIT), ${ }^{17}{ }^{18}$ which was standardised on convalescent stroke patients. The BIT has been shortened and modified for use with acute stroke patients. ${ }^{19}$ The aim of the current study was to evaluate this modified battery as a clinical tool for detecting and monitoring visuo-spatial neglect in patients with an acute stroke.

Method

Forty four consecutive patients with a mean
(SD) age $71.2(12.8)$ years, who had been admitted to hospital with an acute hemispheric stroke, were investigated. Eighteen had a right hemispheric stroke, 26 had a left hemispheric stroke. The diagnosis was confirmed by a physician (SPS) who administered the test battery at three days. Patients were reexamined at three months because after this interval most neurological deficits, including visuo-spatial neglect, made most of their recovery. ${ }^{92021}$ All patients received a non-contrast CT brain scan at three to five days. Two patients had a haemorrhage, the rest had an infarct. In 21 patients $(48 \%)$, the findings on examination were validated against an occupational therapist's assessment of neglect in activities of daily living (ADL). Interobserver reliability was also established using 12 of the stroke patients.

Forty seven age-matched controls were also examined once (see following paper).

\section{The neglect test battery}

All tests were presented in front of the subject's midline with the examiner seated directly opposite. No time limit was imposed. After patients had completed a test they were asked to check that they had finished. Some patients were unable to attempt tests due to the level of consciousness, language difficulties or fatigue. A fundamental feature of patients with visuo-spatial neglect due to a right hemisphere lesion is that they begin tasks on the right hand side. ${ }^{22}{ }^{23}$ In four of the eight tests, the presence of a "Right Hand Start" was assessed. The tests were administered in the following order.

1) POINTING TO OBJECTS LOCATED ABOUT THE WARD $^{24}$ (fig 1) The patient was asked to point to and/or name all the objects that they could see on both sides scattered about their hospital room or ward. The examiner stood directly

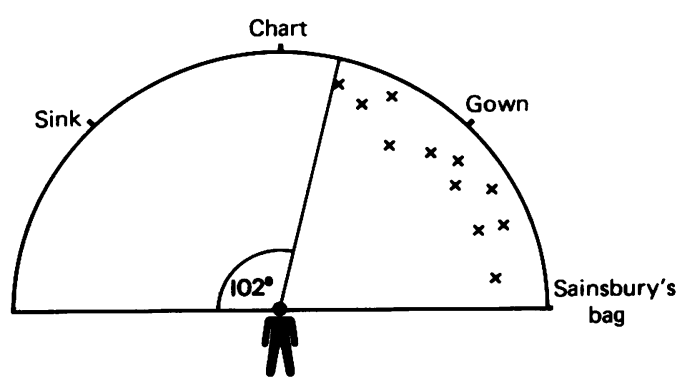

Figure 1 Left visuo-spatial neglect on Pointing to objects. 
Figure 2 Left visuospatial neglect on Menu ( of words underlined), with a right hand start (arrow).

\begin{tabular}{lllll} 
Tomato soup & & Grapefruit & Tea & Roast chicken \\
Pork chop & & Ham \& eggs & hamburger & Lamb chop \\
Shepherd's Pie & Fried haddock & salad & Turkey \\
\hline Jam tart & $\underline{\text { Cheese }}$ & milk & Veal \\
Ice cream & Biscuits & appletart & Boiled eggs \\
Coffee & Apple pie & sandwiches & Melon
\end{tabular}

behind the patient. Using a photocopy of a closed semicircle, with the position of the patient's head marked at the centre of the base and with marks on the circumference at 45,90 and 135 degrees, the examiner noted which objects were situated at $0,45,90,135$ and 180 degrees and used these as landmarks. When the patient pointed to or named objects in the room the examiner marked their approximate position relative to the landmarks and measured the approximate number of degrees omitted.

2) FOOD ON A PLATE ${ }^{17}$ Patients were asked to point to each food item in a lifesize colour photograph of a plate containing eight foods. Where necessary the examiner demonstrated what was required. The total number of items omitted was recorded. Correct verbal identification of the food items was not required.

3) READING A MENU ${ }^{17}$ (fig 2) The patient was asked to open and read aloud from a menu that listed 12 items of food on the left page and 12 on the right. The number of words omitted on the left, right, and in total was recorded. Aphasic patients were allowed to point to words. In a patient with a right hemisphere stroke "Right Hand Start" was defined as a failure to begin reading from the first left hand column ${ }^{25}$ (see arrow on fig 2).

4) READING A NEWSPAPER ARTICLE (adapted from ${ }^{17}$ ) (fig 3) Patients were asked to read the "newspaper" extract aloud. The examiner marked on a photocopy any words omitted. Some patients were unable to read small print, so the number of words omitted from the headlines (10 words in bold print), the paragraph (five words in smaller capitals) and

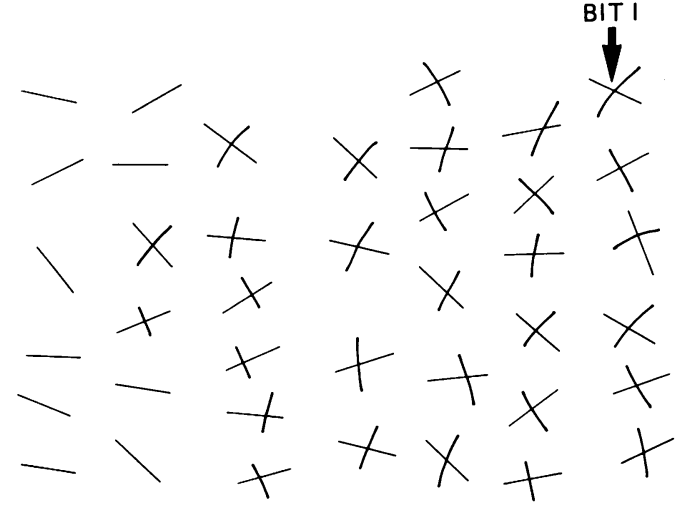

Figure 4 Left visuo-spatial neglect on Line cancellation with a Right Hand Start (arrow).

the article (117 words in small newsprint) were recorded separately. The presence or absence of "Right Hand Start" was recorded. This test was not given to patients with a left hemisphere stroke because the pilot study ${ }^{19}$ that modified the BIT found that most of them had reading difficulties.

5) LINE CANCELLATION (adapted from ${ }^{17} 27$ ) (fig 4) The patient was presented with a sheet of paper on which 40 one inch lines had been marked in seven columns. The patient was required to cross out all the lines on the page after the examiner had demonstrated what was required by crossing out the four lines in the centre column. A "Right Hand Start" was considered present if the first lines to be cancelled were in the sixth or seventh columns on the right of the page (see arrow in fig 4 ). The number of lines omitted on the right, the left and in total was recorded. If the patient's dominant hand was too weak to cross out lines and they were unable to use the other hand, they were allowed to point to each line, which the examiner then crossed out.

6) STAR CANCEllation ${ }^{17}$ (fig 5) Patients were presented with 56 small stars mixed up with many large stars and capital letters. They were instructed to cross out the small stars after the examiner had demonstrated this, crossing out two centrally positioned small stars. The number of small stars omitted on the left, the right and in total was recorded. A "Right Hand Start" was considered present if the

\section{Driver killed, 30 hurt as packed train rams a loco}
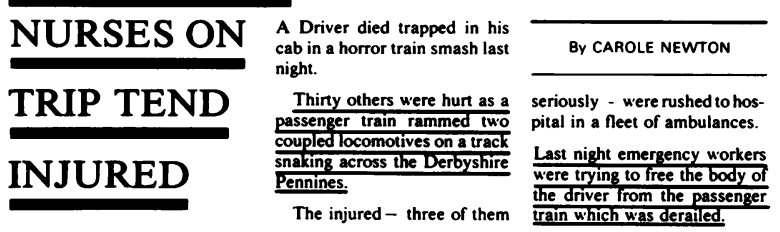

More than 100 people were on the four-coach diesel when it crashed near the village of Chinkey near Buxton.

The alarm was raised by a young motor-cyclist who saw the crash and ran to a nearby school for help.

Two nurses on board the train

Figure 3 Left visuo-spatial neglect on the Newspaper (of words underlined).

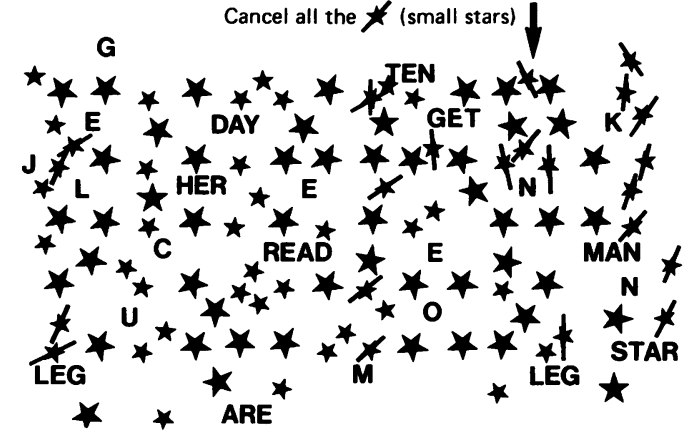

Figure 5 Left visuo-spatial neglect on Star cancellation, with a Right Hand Start. 


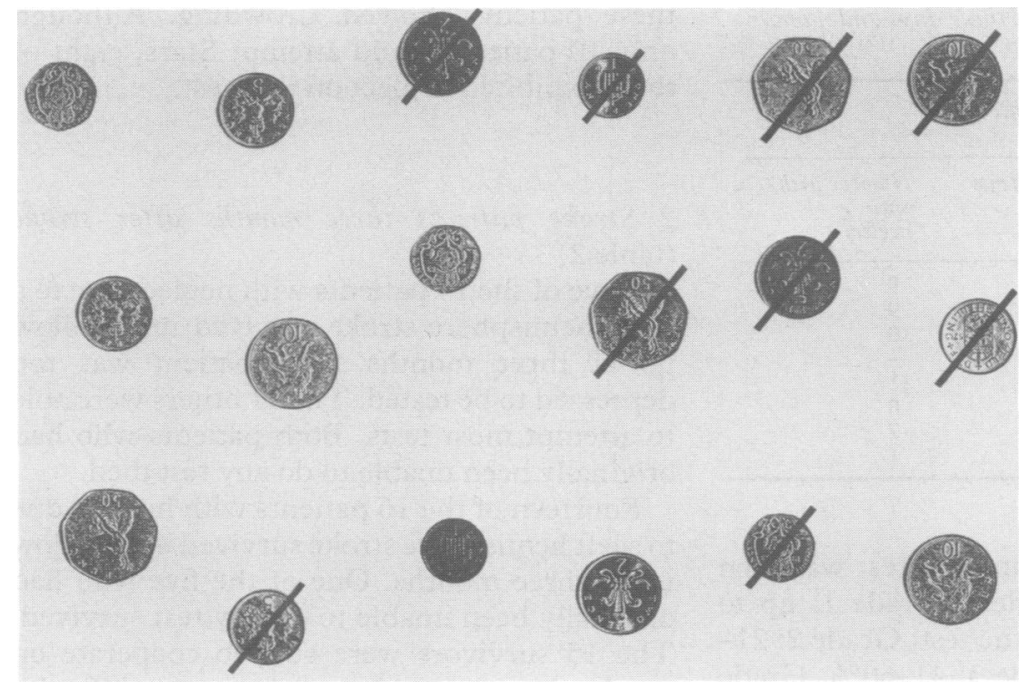

Figure 6 Left visuo-spatial neglect on Coin selection (unmarked coins).

patient began cancelling stars in the right hand third of the page. Patients with a weak dominant hand were allowed to point to each star.

7) COIN SELECTION ${ }^{17}$ (fig 6) A large card $(32 \times 21 \mathrm{~cm})$ with three coins of each of the following values $(2 \mathrm{p}, 5 \mathrm{p}, 10 \mathrm{p}, 20 \mathrm{p}, 50 \mathrm{p}, £ 1)$ was presented to the patient with the three five pence coins on the side opposite their cerebral lesion. The patient was asked to point to each of the coins in turn. The examiner noted the results on a photocopy of the test card. The number of omissions on the left, right and in total was recorded. Occasionally the examiner placed a loose coin of the value required in front of aphasic patients or wrote the value of the coin on a piece of paper to help them understand which coin was required.

Figure 7 Right visuospatial neglect on Figure copying with Crowding.

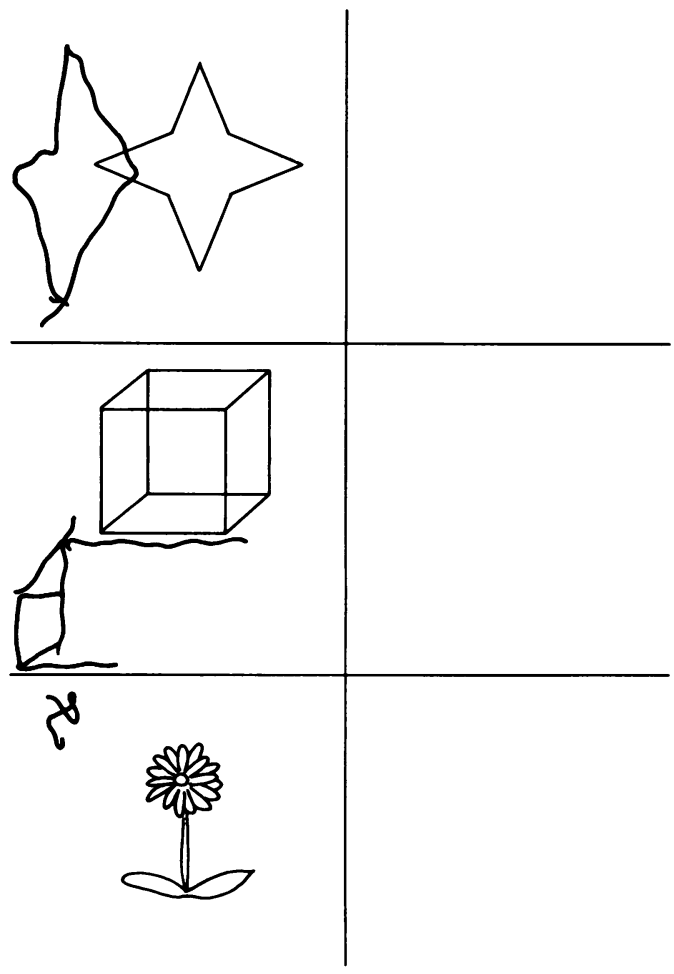

8) FIGURE COPYING FROM THE LEFT ${ }^{17}$ (fig 7) The patient was presented with a piece of paper divided into six squares. In the three squares on the left were figures of a four pointed star, ${ }^{7}$ a cube ${ }^{28}$ and a daisy. ${ }^{29}$ These were to be copied into the empty three spaces on the right side. The number of major omissions (for example, half a cube) and the number of minor omissions (for example, a leaf) was recorded for each figure. Each major omission was given an arbitrary score of two and each minor omission a score of one.$^{30}$ This test was not given to those patients with a right hemisphere stroke as the pilot study that modified the $\mathrm{BIT}^{19}$ indicated that this test was less sensitive than many others. It proved useful, however, for patients with left hemisphere stroke who often ignored the empty right spaces and "crowded" their copies into the left hand side of the page.

\section{CRITERIA FOR VISUO-SPATIAL NEGLECT}

To establish a normal range of performance for each test the controls were examined; these results are given in an accompanying paper. Visuo-spatial neglect was considered present in patients if they made more omissions on any one test than the age-matched controls. Thus two or more omissions on meal; one or more on menu; five or more on lines; 16 or more on stars; four or more on coins; two or more on headlines; two or more on paragraph; seven or more on article; and 50 or more degrees on pointing was regarded as evidence of neglect. Major or minor omissions on left figure copying, Crowding and a Right Hand Start on the reading tests never occurred in controls and were thus considered clinical indicators of neglect.

In addition, any stroke patient making three unilateral omissions on coin sorting was considered to have neglect on that test if neglect was also present on another test. Similarly, neglect was considered present in star cancellation when a stroke patient omitted between six and 15 stars if there were at least twice as many omissions on one side of the page as the other ${ }^{4}$ and if neglect was present in another test. Also, a Right Hand Start on line and star cancellation was taken as indication of neglect if neglect was present on these or other tests. Combinations of these features were not observed in the controls.

\section{SCORING THE TESTS}

The total number of omissions made on lines, stars, meal, menu and coins was expressed as a percentage of the total number of items in each test. ${ }^{4}$ The number of degrees omitted on pointing was expressed as a percentage of 180 degrees. The number of words omitted in headlines was expressed as a percentage out of 10, the number omitted on Paragraph as a percentage of five, and the number in the Article was expressed as a percentage of 117 . The score for figure copying was converted into a percentage (of a maximum score of six). 
Table 1 Number of stroke patients able to attempt each test at three days and number with neglect on each test

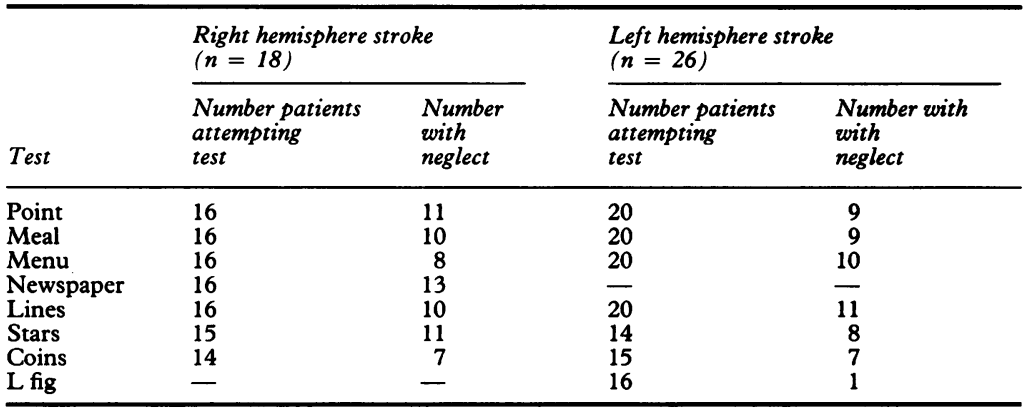

The percentage score on each test was then graded: Grade 0: no neglect; Grade 1: up to $20 \%$ of items omitted on the test; Grade 2: $21-$ $40 \%$ items omitted; Grade 3: $41-60 \%$; Grade 4: $61-80 \%$; Grade 5: $81-100 \%$. Right Hand Start and Crowding were both considered to be qualitative rather than quantitative measures of neglect and were noted but not scored.

\section{Results}

1 Stroke patients three days after stroke (table 1) At three days after stroke, two patients with a right hemisphere stroke could not be assessed on any test because of coma. All remaining 16 patients could be tested on Pointing, Meal, Menu, Newspaper and Lines. One patient was too tired to do stars or coins, another could not recognise coins.

Five patients with a left hemisphere stroke could not be assessed at three days because of coma or severe aphasia. Most of the remaining 21 could be assessed on Pointing, Meal, Menu and Lines. Aphasia made it impossible for some patients to attempt Stars, Coins and Figure copying because they were unable to understand the instructions.

At three days $13 / 18(72 \%)$ patients with a right hemisphere stroke showed neglect on at least one test. Neglect was present in 16/26 $(62 \%)$ patients with a left hemisphere stroke.

The tests most likely to detect neglect in a patient with a right hemisphere stroke were Newspaper, Stars, Pointing, Meal and Lines (table 1). Eleven of these patients demonstrated a Right Hand Start.

For patients with a left hemisphere stroke the most likely tests to detect neglect were Meal, Menu, Lines and Pointing (table 1). Five of

Table 2 Number of stroke patients able to attempt each test at three months and number with neglect on each test

\begin{tabular}{llllll}
\hline & \multicolumn{2}{l}{$\begin{array}{l}\text { Right hemisphere stroke } \\
(n=12)\end{array}$} & & $\begin{array}{l}\text { Left hemisphere stroke } \\
(n=15)\end{array}$ \\
\cline { 2 - 3 } \cline { 5 - 6 } Test & $\begin{array}{l}\text { Number patients } \\
\text { attempting } \\
\text { test }\end{array}$ & $\begin{array}{l}\text { Number } \\
\text { with } \\
\text { neglect }\end{array}$ & & $\begin{array}{l}\text { Number patients } \\
\text { attempting } \\
\text { test }\end{array}$ & $\begin{array}{l}\text { Number with } \\
\text { with } \\
\text { neglect }\end{array}$ \\
\hline Pointing & 11 & 3 & & 15 & 2 \\
Meal & 11 & 6 & & 15 & 3 \\
Menu & 11 & 3 & & 15 & 3 \\
Newspaper & 11 & 5 & & 15 & 3 \\
Lines & 11 & 7 & & 12 & 3 \\
Stars & 11 & 5 & & 12 & 0 \\
Coins & 11 & - & 15 & 3 \\
L fig & - & &
\end{tabular}

these patients showed Crowding. Although only 10 patients could attempt Stars, eight of these exhibited neglect on that test.

2 Stroke patients three months after stroke (table 2)

Twelve of the 13 patients with neglect due to a right hemisphere stroke survived until follow up at three months. One patient was too depressed to be tested. The 11 others were able to attempt most tests. Both patients who had originally been unable to do any test died.

Fourteen of the 16 patients with neglect due to a left hemisphere stroke survived until follow up at three months. One of the five who had originally been unable to do any test survived. The 15 survivors were able to cooperate on nearly every test although language difficulty still prevented some patients understanding the Coins and Stars test requirements.

Neglect was present in nine patients with a right hemisphere stroke $(75 \%)$ and five left hemisphere stroke patients $(33 \%)$.

In those with a right hemisphere stroke the tests most likely to detect neglect were Stars, Meal, Coins and Newspaper. A Right Hand Start was present in nine of these patients. In patients with a left hemisphere stroke the tests most likely to detect neglect were Menu, Stars and Coins.

\section{Validity of the test battery}

To ascertain the validity of the test battery an occupational therapist blind to the results of the battery carried out an assessment on 21 of the patients, using the following checklist of "neglect behaviours".

1) Did the patient fail to orientate to environmental stimuli on the side opposite the cerebral lesion?

2) Did the patient fail to dress, wash or groom their contralateral side?

3) Did the patient bump into doorways or obstacles on the contralateral side, when walking or using a wheelchair? If using the latter, were they unable to turn contralaterally? If mobile, did the patient veer diagonally towards the contralateral side?

4) Could any failure or reluctance to use the contralateral limbs be attributed to neglect rather than weakness or apraxia? ${ }^{31}$

5) Did the patient show lack of awareness of limbs with poor positioning? ${ }^{31}$

Four patients (two with a right, two with a left hemisphere stroke) had no neglect on the battery and none on the occupational therapist's checklist. The remaining 17, (12 with a right, five with a left hemisphere stroke), had visuo-spatial neglect on the test battery. Thirteen of these had neglect on at least three checklist items, two had neglect on two items (orientation, washing/dressing), and one neglected on one item (orientation). One patient with marked left visuo-spatial neglect on the battery, a left homonymous hemianopia and no hemiparesis, had no neglect on the checklist. 
Table 3 Severity of neglect in 11 patients with a right hemisphere and in 14 patients with a left hemisphere stroke at three days and at three months after stroke

\begin{tabular}{|c|c|c|c|c|}
\hline \multirow[b]{2}{*}{ Test } & \multicolumn{2}{|c|}{$\begin{array}{l}\text { Right hemisphere stroke } \\
\text { Median score }\end{array}$} & \multicolumn{2}{|c|}{$\begin{array}{l}\text { Left hemisphere stroke } \\
\text { Median score }\end{array}$} \\
\hline & 3 days & 3 months & 3 days & 3 months \\
\hline $\begin{array}{l}\text { Pointing } \\
\text { Meal } \\
\text { Menu } \\
\text { Lines } \\
\text { Stars } \\
\text { Coins } \\
\text { Headlines } \\
\text { Paragraph } \\
\text { Article } \\
\text { L fig }\end{array}$ & $\begin{array}{l}3.0 \\
3 \cdot 0 \\
3 \cdot 0 \\
4 \cdot 0 \\
3.0 \\
3 \cdot 0 \\
1.0 \\
4 \cdot 0 \\
2 \cdot 0 \\
-\end{array}$ & $\begin{array}{l}0.0 \\
1.0 \\
0.0 \\
0.0 \\
2.0 \\
2.0 \\
0.0 \\
0.0 \\
0.0 \\
-\end{array}$ & $\begin{array}{l}1.0 \\
0.0 \\
2.0 \\
2.0 \\
3.0 \\
2.0 \\
= \\
= \\
0.0\end{array}$ & $\begin{array}{l}0.0 \\
0.0 \\
0.0 \\
0.0 \\
0.0 \\
0.0 \\
= \\
= \\
0.0\end{array}$ \\
\hline
\end{tabular}

\section{Reliability of the test battery}

Inter-observer reliability was established by the simultaneous scoring of 12 patients (eight with a right and four with a left hemisphere stroke) with neglect by two examiners trained in the administration of the battery.

Each patient was assessed on nearly every test, although only 11 were able to do Pointing, Meal, Menu and Stars. There was agreement as to the presence or absence of neglect in every test except in Coins in one patient to whom the newer coins may have been unfamiliar. Both observers agreed on the graded score on each test except in Newspaper in one patient who mumbled inaudibly.

\section{Sensitivity to changes in neglect}

Table 3 shows the median test scores of those patients presenting with neglect at three days after stroke who survived and were able to be examined at three months. There were 11 with a right and 14 with a left hemisphere stroke.

At three days neglect was greater in patients with a right than in patients with a left hemisphere stroke, but the difference only reached significance for Lines ( $p=0.04$; Mann-Whitney tests). At three months neglect had declined on all tests in right hemisphere patients. This was significant at the 0.02 level for Lines, Stars, and Pointing; and at the 0.05 level for Paragraph. Neglect also declined in left hemisphere strokes. This was significant at the 0.01 level for Lines, and at the 0.05 level for Pointing, Meal, Menu, and Stars (Wilcoxon tests).

\section{Discussion}

The study describes the use of a test battery to detect, measure and monitor visuo-spatial neglect. It is relevant, valid, reliable, simple to administer, sensitive and gives a result that can be communicated to fellow professionals. ${ }^{32}$

The battery includes tests from the BIT which simulate performance in everyday activities, for example, reading, looking around the room, selecting coins, and eating. These tests would seem more relevant to real life and may indicate to therapists appropriate areas for treatment. ${ }^{1718}$ The validity study showed that 16 out of 17 patients with neglect on the battery, had neglect on the OT checklist. This method of validating clinical tests of neglect has been used by Battersby et al who found that neglect on clinical testing did not always generalise to daily activities. ${ }^{233} \mathrm{We}$ observed one such patient, but cannot say whether such a discrepancy is an example of a dissociation between neglect in different tasks. ${ }^{15}$

In 12 patients assessed by two observers, the reliability of the battery was good with respect to the presence and severity of neglect in each test. The tests were easy to administer. The presence of aphasia was not as great an obstacle to assessment as had been anticipated for sufficient gestural comprehension was often retained. Seven consecutive patients whose performance was timed completed the battery in a mean (SD) time of 11 minutes 10 seconds ( 3 minutes 11 seconds).

The battery was sensitive to the presence of visuo-spatial neglect, and to changes in its severity over time. At three days the incidence of visuo-spatial neglect was $72 \%$ (right hemisphere) and $62 \%$ (left hemisphere). At three months the incidence in survivors was $75 \%$ (right hemisphere) and 33\% (left hemisphere). Other studies which have used a simple line cancellation and figure copying battery ${ }^{46}$ have reported a lower incidence of visuo-spatial neglect in acute stroke. Had Line cancellation been used as the sole test of neglect the incidence of neglect would have been $55 \%$ (right hemisphere) and $42 \%$ (left hemisphere) at three days after stroke. At three months the incidence of neglect would have been only $33 \%$ (right hemisphere) and $0 \%$ (left hemisphere). The likely reason for our higher incidence of neglect is that we used a larger battery and considered neglect to be present when it appeared in a single test. ${ }^{30}$ Neglect may be taskspecific. ${ }^{13-15}$ Assessment of patients by a larger battery is therefore more likely to result in the detection of visuo-spatial neglect than assessment by a one or two test battery. ${ }^{30}$ The use of a larger battery has a particular bearing on the detection of neglect in left hemisphere stroke. Although some patients were unable to understand what was required in some tests, they often understood and attempted others. This may be why neglect was nearly as common after left hemisphere stroke as after right. Other workers have found this ${ }^{27}{ }^{3034}$ but the classic view is that neglect is associated almost solely with right brain damage. ${ }^{35}$

There is no one standard method of measuring neglect. ${ }^{417183036}$ Some sensitive indicators in our battery are not scorable on an ordinal scale, for example Right Hand Start and Crowding but they remain useful clinical signs of neglect, possibly reflecting abnormalities in eye movements reported in patients with unilateral brain damage who begin visual search tasks by exploring ipsilesional space. ${ }^{22}{ }^{34}$ Expressing the percentage of items omitted from each test as a grade on a $0-5$ scale is simple and each grade is easily understood. It avoids giving too much weight to small changes over time in the raw score, expresses the result of each test in the same arbitrary units and enables change to be monitored. Our observation that neglect is more severe in those with a right than in those with a left hemisphere stroke is consistent with other reports. ${ }^{22} 34$ 
In conclusion, the battery takes into account the behavioural inconsistency of neglect and is suitable for use with patients after an acute stroke.

SPS was supported by a grant from the Chest Heart and Stroke Association. We are grateful to Dr J R Jenner, Consultant in Rehabilitation, and in particular to Dr CM C Allen, Consultant Neurologist, Addenbrookes, Cambridge for early discussions; and also the library staff of the Royal Society of Medicine.

and also the library staff of the Royal Society of Medicine. Thames Valley Test Company.

1 Feigenson JS, Mccarthy ML, Greenberg SD, Feigenson WD. Factors influencing outcome and length of stay in stroke rehabilitation unit. Part 2. Comparison of 318 screened and 248 unscreened patients. Stroke 1977;8: $657-62$.

2 Kinsella G, Ford B. Acute recovery patterns in stroke patients. Neuropsychological factors. Med J Australia 1980;2:663-6.

3 Denes G, Semenza C, Stoppa E, Lis A. Unilateral spatial neglect and recovery from hemiplegia. A follow up study. Brain 1982;105:543-52.

4 Fullerton KJ, Mackenzie G, Stout RW. Prognostic indices in stroke. $Q J$ Med 1988;66:147-62

5 Adams GF, Hurwitz LJ. Mental barriers to recovery from strokes. Lancet 1963;2:532-7.

6 Cutting J. The study of anosognosia. J Neurol Neurosurg Psychiatry 1978;41:548-55.

7 Oxbury JM, Campbell DC, Oxbury SM. Unilateral spatial neglect and impairment of spatial analysis and visua perception. Brain 1974;97:551-64.

8 Hier DB, Mondlock J, Caplan LR. Behavioural abnormalities after right hemisphere stroke. Neurology 1983;33:337-44

9 Hier DB, Mondlock J, Caplan LR. Recovery of behavioural abnormalities after right hemisphere stroke. Neurology 1983;33:345-50.

10 Campbell DC, Oxbury SM. Recovery from unilateral spatial neglect? Cortex 1976;12:303-12.

11 Mesulam M-M. A cortical network for directed attention and unilateral neglect. Ann Neurol 1981;10:309-25.

12 Friedland RP, Weinstein EA. Hemi-inattention and hemisphere specialization: introduction and historical review. Advances in Neurology 1977;18:1-31.

13 Leicester J, Sidman M, Stoddard LT, Mohr JP. Some determinants of visual neglect. J Neurol Neurosurg Psychiatry 1969;32:580-7.

14 De Lacy Costello A, Warrington EK. The dissociation of visuo-spatial neglect and neglect dyslexia. $J$ Neurol Neurosurg Psychiatry 1987;50:1110-6.

15 Bisiach E, Perani D, Vallar G, Berti A. Unilateral neglect: personal and extra-personal. Neuropsychologic 1986;24:759-67.

16 Andrews K, Brocklehurst JC, Richards B, Laycock PJ. The prognostic value of picture drawings by stroke patients. Rheum Rehabil 1980;19:180-8.
17 Wilson B, Cockburn J, Halligan P. Development of a behavioural test of visuo-spatial neglect. Arch Phys Med 1987;68:98-102.

18 Wilson B, Cockburn J, Halligan P. The Behavioural Inattention Test. Fareham, Hampshire: Thames Valley Test

19 Stone SP, Wilson B, Rose FC. The development of a standard test battery to detect, measure and monitor visuo-spatial neglect in acute stroke. Int $J$ Rehab Res 1987;10:110.

20 Skilbeck CE, Wade DT, Hewer RL, Wood VA. Recovery after stroke. J Neurol Neurosurg Psychiatry 1983;46:5-8.

21 Allen CMC. Predicting the outcome of acute stroke: a prognostic score. J Neurol Neurosurg Psychiatry $1984 ; 47: 475-80$.

22 Chedru F, Leblanc M, L'Hermitte F. Visual searching in normal and brain-damaged subjects (a contribution to the study of uni-lateral attention). Cortex 1973;9:94-111.

23 Weinburg J, Diller L, Gordon WA, Gerstmann LJ, Liebermann A, Lakin $P$, et al. Visual scanning training effect on reading related tasks in acquired right brain damage. Arch Phys Med Rehabil 1977;58:479-86.

24 Patterson A, Zangwill O. Disorders of visual space perception associated with lesions of the right cerebral hemisphere. Brain 1944;67:331-58.

25 Mesulam MM. The functional anatomy and hemispheric specialization for directed attention. The role of the parietal lobe and its connectivity. Trends Neurosci

26 Holmes G. Disturbances of visual orientation. Br JOphthalmol 1918;2:449-68.

27 Albert ML. A simple test of neglect. Neurology 1973;23: 658-64.

28 Gainotti G, Messerli P, Tissot R. Qualitative analysis of unilateral spatial neglect in relation to laterality of cerebral lesions. J Neurol Neurosurg Psychiatry 1972;35:545-50.

29 Gregory ME, Aitken JA Assessment of parietal lobe function in hemiplegia. Occupational Therapy 1971:34:9-17.

30 Ogden JA. Anterior-posterior interhemispheric differences in the loci of lesions producing visual hemi-neglect. Brain and Cognition 1985;4:59-75.

31 Heilman KM, Watson RT, Valenstein E Neglect and related disorders. In: Heilman KM, Valenstein E, eds. related disorders. In: Heilman KM, Valenst

32 Wade DT, Hewer RL, Skilbeck CE, David RM, eds. Stroke. A critical approach to diagnosis, treatment and management, London: Chapman and Hall, 1985

33 Battersby WS, Bender MR, Pollack M, Kahn RL. Unilateral "spatial agnosia" ("inattention") in patients with cerebral lesions. Brain 1956;79:68-93.

34 Chain F, Leblanc M, Chedru F, L'Hermitte F. Negligence visuelle dans les lesions posterieures de l'hemisphere gauche. Rev Neurol 1979;135:105-26.

35 Haecen $\mathrm{H}$. Clinical symptomatology of right and left hemisphere lesions. In: Mountcastle VB, ed. Interhemispheric ( Press, 1962.

36 Wade DT, Wood VA, Hewer RL. Recovery of cognitive function soon after stroke; a study of visual neglect, function soon after stroke; a study of visual neglect,
attention span and verbal recall. J Neurol Neurosurg Psychiatry 1988;51:10-13. 